\title{
The $\mathbf{R}^{*}$-operation and five-loop calculations
}

\author{
Ben Ruijl* \\ Institute for Theoretical Physics, ETH Zurich, Wolfgang-Pauli-Str. 27, 8093 Zürich, CH \\ E-mail: bruijleethz.ch
}

Franz Herzog, Takahiro Ueda, Jos Vermaseren

Nikhef Theory Group, Science Park 105, 1098 XG Amsterdam, NL

E-mails: fherzog@nikhef.nl, tueda@nikhef.nl, t68@nikhef.nl

\section{Andreas Vogt}

Department of Mathematical Sciences, University of Liverpool, Liverpool L69 3BX, UK

E-mail: Andreas.Vogtaliv.ac.uk

\begin{abstract}
We sketch how the $R^{*}$-operation can be used to compute the pole terms of Feynman diagrams. We identify computational difficulties when performing five-loop calculations, and provide four solutions that drastically reduce the number of terms that are generated. Using these methods, we have computed the beta function for Yang-Mills theory with fermions, the $R$-ratio in electronpositron annihilation, and Higgs decays to quarks and gluons at five-loop accuracy. The results for the beta function and Higgs decay width to gluons in the heavy-top limit are briefly discussed. There is no need for six-loop extensions of these calculations in the near future.
\end{abstract}

13th International Symposium on Radiative Corrections 24-29 September 2017, St. Gilgen, Austria

${ }^{*}$ Speaker. 


\section{Introduction}

Many physically interesting quantities can be computed from the pole parts of Feynman diagrams, for example, anomalous dimensions (such as the beta function), splitting functions, and decay rates. Often, it is much easier to compute the pole parts of diagrams than the finite part.

In this work we sketch the $R^{*}$-operation [1-3], which is able to compute the pole parts of $L$ loop diagrams by a computation of at most $(L-1)$-loop diagrams. Using a high-performance implementation of the $R^{*}$-operation combined with the FORCER program [4-6], we are able to compute the poles of five-loop massless propagator diagrams. Our programs rely heavily on FORM [7-10].

Performing efficient computations of five-loop diagrams is hard, since the Feynman rules create many terms. To alleviate these issues, we describe four methods to reduce the number of terms which are not specific to the $R^{*}$-method: (1) removal of propagator insertions, (2) delaying of Feynman rule substitution, (3) canonicalization of Feynman diagrams, and (4) efficient tensor reduction.

Using these optimizations, the five-loop beta function for Yang-Mills theory with fermions [11] can be computed in six days on one 32-core machine. We have also computed the $R$-ratio in $e^{+} e^{-} \rightarrow$ hadrons, and the Higgs decays to bottom quarks and gluons at five loops [12].

The outline of this paper is as follows. In section 2 we very briefly describe the $R^{*}$-operation. In section 3 we address the four optimizations. In section 4 we briefly discuss the above physics results. Finally, we summarize and present a brief outlook in 5 .

\section{The $R^{*}$-operation}

The $R^{*}$-operation can be used to compute the poles of Feynman diagrams [1,2]. Recently, it has been extended to Feynman diagrams with arbitrary numerator structure [3]. In this section we briefly sketch how the $R^{*}$-operation works, focusing on UV-counterterms.

The basic object of the $R^{*}$-operation is the UV counterterm operation $\Delta$ acting on a graph $G$, which is defined as the poles of $G$ in the limit of all loop momenta going to infinity with all contributions from subdivergences subtracted. Additionally, we define the pole operator $K$ for a Laurent series in $\varepsilon$

$$
K \sum_{i=-\infty}^{\infty} c_{i} \varepsilon^{i}=\sum_{i=-\infty}^{-1} c_{i} \varepsilon^{i} .
$$

Then the $R^{*}$ operation for some simple examples yields

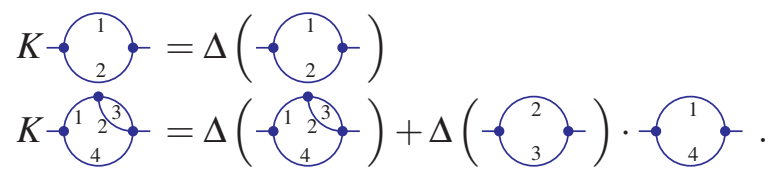

In general, all sets of non-overlapping divergent subdiagrams have to be considered:

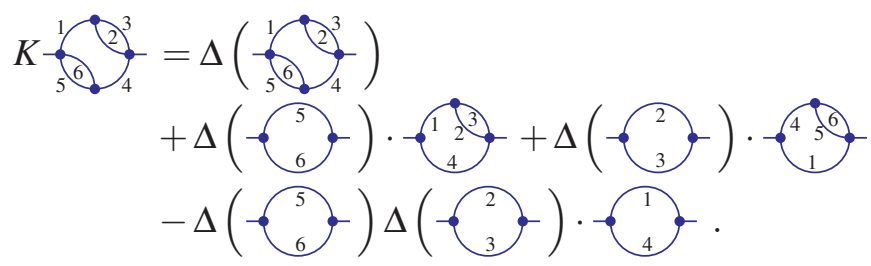

Here the contribution from two counterterms gets a minus sign, to prevent double counting. 
For logarithmically divergent diagrams, $\Delta$ does not depend on external momenta or masses. Consequently, we can infrared rearrange (IRR) [13]:

$$
\Delta(\circlearrowleft)=\Delta(\longmapsto)=\Delta(\longmapsto) \text {. }
$$

Using IRR and the definition of $\Delta$, we can express the UV-counterterm of $G$ in terms of simpler diagrams:

$$
\Delta(G) \stackrel{\text { IRR }}{=} \underbrace{\Delta\left(G^{\prime}\right)}_{\text {Simpler than } \mathrm{G}}=K\left(G^{\prime}\right)-\underbrace{\text { subdivergences }\left(G^{\prime}\right)}_{\text {Lower-loop diagrams }}
$$

Using this setup, we can rewrite all $L$-loop diagrams to $(L-1)$-loop scalar massless propagator integrals. For five-loop applications, all those integrals can be computed using FORCER [5,6].

\section{Optimisations}

Performing computations at five loops introduces at least four new bottlenecks compared to four loops: (1) the number of diagrams and their complexity grow exponentially, (2) the substitution of the Feynman rules is slow and creates millions of terms, (3) the number of counterterms grows exponentially, and (4) tensors of rank 10 have to be reduced, which involves solving large systems.

In this section we address these issues by presenting five optimisations, namely improved treatment of propagator insertions in section 3.1, delayed Feynman rule substitution in section 3.2, a canonical form algorithm for Feynman diagrams in section 3.3, and an efficient tensor reduction algorithm in section 3.4.

\subsection{Treatment of propagator insertions}

Many of the higher-loop corrections are self-energies of propagators in the diagram. Due to the local nature of the Feynman rules, these self-energies only depend on their external momentum (there are no contractions with other parts of the larger diagram), so they can be 'factorised' out,

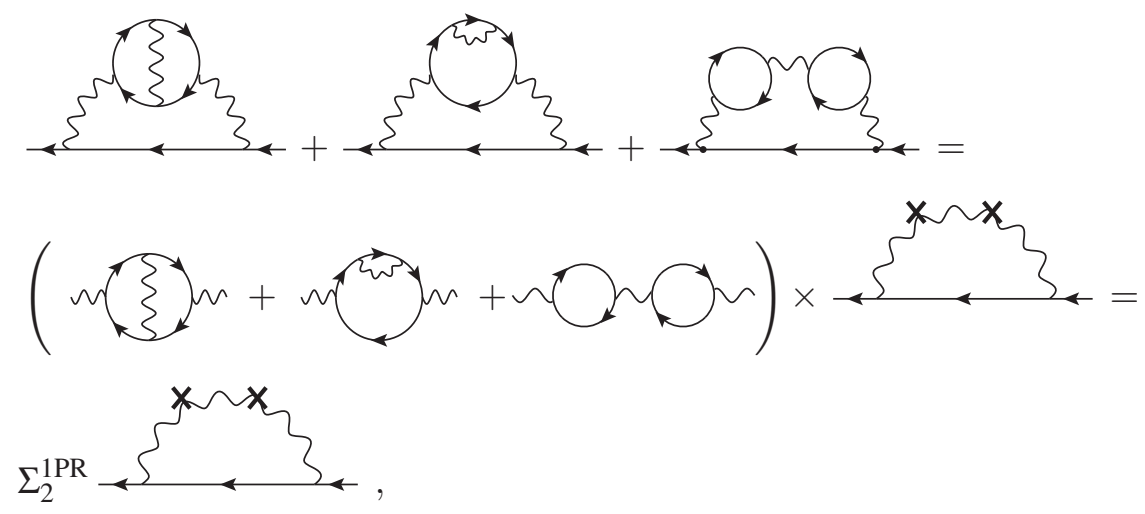

where the $L$-loop self-energy is replaced by $\left(p^{2}\right)^{-\varepsilon L}$ in the larger diagram (marked by $L$ crosses). In a sense, the subdiagram is integrated out. The resulting simpler topology is multiplied by the one-particle-reducible $L$-loop self-energy. Since the $L$-loop subdiagram is of lower order, these 
quantities have already been computed and can easily be tabulated to prevent recomputations. For example, a five-loop diagram may contain the expensive 4-loop gluon propagator as a subdiagram.

For the $R^{*}$-operation, this representation has an issue: the non-integer power hides UV-divergent subdiagrams, which should be subtracted. However, since the exact contents of the $\left(p^{2}\right)^{-\varepsilon L}$ is factorised out, we may replace it with any $L$-loop subdiagram. Therefore we choose the simplest configuration: $L$ scalar one-loop bubbles side by side. Thus, for the $R^{*}$-operation we can remove propagator insertions by using the following relation:

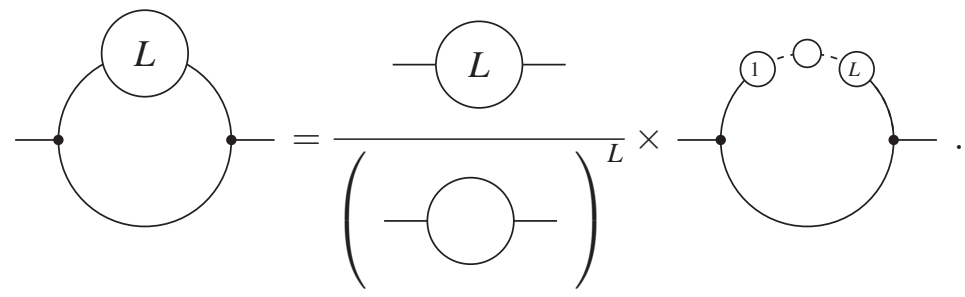

\subsection{Delayed Feynman rule substitution}

Substituting the Feynman rules creates many terms. For example, the following fully gluonic fiveloop graph creates 12029521 scalar integrals in the Feynman gauge:

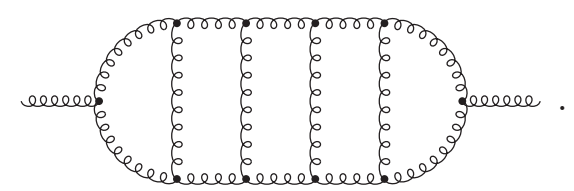

The source of the blow-up is the Feynman rule for the triple gluon vertex, which can be written in the following way:

$$
v_{3 g}\left(p_{1}^{\mu, a}, p_{2}^{v, b}, p_{3}^{\rho, c}\right)=-i f^{a b c}\left[\left(p_{1}-p_{2}\right)^{\rho} g_{\mu v}+\left(2 p_{2}+p_{1}\right)^{\mu} g_{v \rho} x+\left(-2 p_{1}-p_{2}\right)^{v} g_{\mu \rho}\right] .
$$

Thus, for every vertex, six terms are created, of which some will evaluate to the same expression due to symmetries. For all these terms, expensive operations such as Taylor expansions and divergent subgraph recognitions have to be performed. However, these operations only depend on the momentum powers and are invariant under the way the momenta contract. So, we rewrite the triple gluon vertex in a way that exposes the momenta, but keeps all the contractions unsubstituted:

$$
v_{3 g}\left(p_{1}^{\mu, a}, p_{2}^{v, b}, p_{3}^{\rho, c}\right)=-i f^{a b c} p_{1}^{\sigma} t_{3}(\sigma, v, \rho, \mu)+i f^{a b c} p_{2}^{\sigma} t_{3}(\sigma, \mu, \rho, v),
$$

where

$$
t_{3}(\mu, v, \rho, \sigma)=g_{\mu \rho} g_{v \sigma}+g_{\mu \sigma} g_{v \rho}-2 g_{\mu v} g_{\rho \sigma} .
$$

After rewriting $v_{3 g}$ in terms of $t_{3}$, there are only $2^{10}=1024$ terms for the Feynman diagram in eq. (3.3). We can keep our input in this compactified notation for as long as the actual contractions are not important, which is right until the tensor reduction.

We define the operation $\circ$ that applies the remaining Feynman rules to all components of the $R^{*}$-operation, for example,

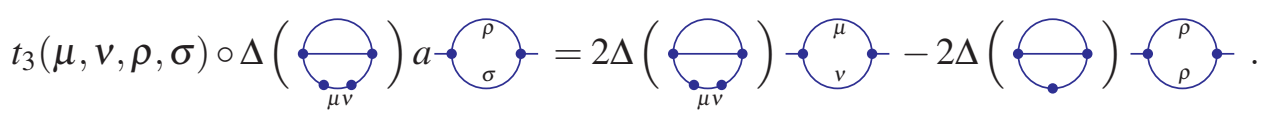


We stress that for this particular case contraction is necessary.

Similar rules can be devised for the other vertices and for the trace of gamma matrices. At five loops, the substitution of $t_{3}$ and similar structures is an expensive part of the calculation, since the number of generated terms is high.

\subsection{Canonical forms for Feynman diagrams}

The $R^{*}$-operation applied to five-loop diagrams will create many counterterms. In order to reduce computation time, it is important to compute the counterterms of a specific graph only once. In turn, this requires an efficient way to detect if two graphs are equal. One straightforward option is to keep a list of all the graphs that have already been processed and test for isomorphisms on every element of the list until one is found. If no match is found, the current graph can be added to the list. The two downsides of this method are that (1) an isomorphism test can be rather slow at five loops and (2) that the list of topologies grows rapidly.

A better alternative is to construct a canonical form of a graph. A canonical form is an isomorphism of the graph that is designated as the smallest by some yet to be defined measure. To test for equality, one can simply compare the canonical forms. Since isomorphy is first and foremost a property of the vertices, we give each vertex a label from 1 to $n$. For simplicity, let us consider a graph that has no dot products and only has edges with power 1.

We convert our graph to an edge representation:

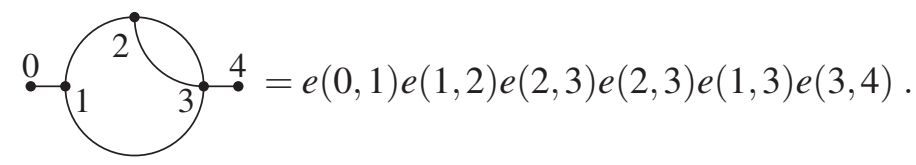

Here, $e\left(n_{1}, n_{2}\right)$ is the edge function, in which we place the smallest vertex index as the first argument. The edge list is a lexicographically sorted list of edge functions, as is shown in eq. (3.8). Now we define the smallest isomorphism of a graph as the vertex labeling for which the edge list is lexicographically smallest. ${ }^{1}$

We can easily extend the graph notation to a graph where propagators can have different powers, by introducing a third argument to the edge function $e$ :

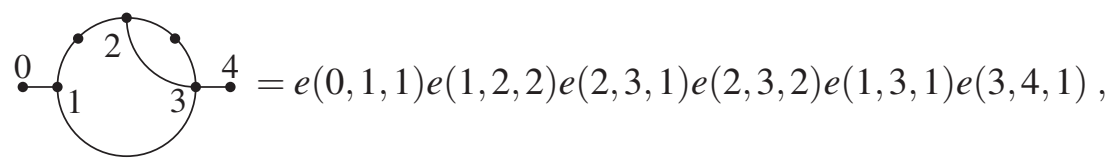

where we again make sure that the first two arguments of $e\left(n_{1}, n_{2}, \ldots\right)$ are sorted. To add support for dot products and tensors, we extend the edge function even further:

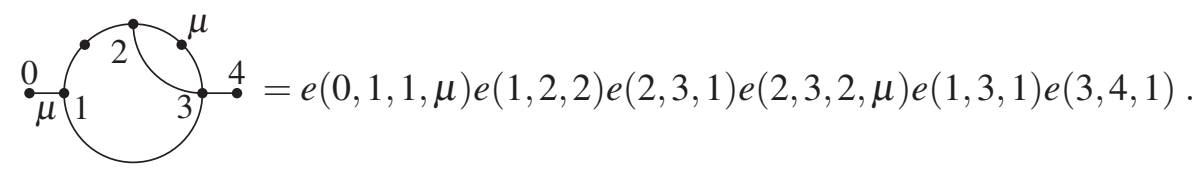

\footnotetext{
${ }^{1}$ In our program, we use the internal (deterministic) sorting order of FORM [7-9] to determine the smallest isomorphism instead. The latest version of FORM [10] is required for our $\mathrm{R}^{*}$ program.
} 
We define the canonical signs of the momenta such that they always flow from the smallest vertex label to the highest. If a transformation changes the order, we flip the sign if the number of vectors in the momentum is odd:

$$
e\left(2,1, n, \mu_{1}, \ldots, \mu_{k}\right)=(-1)^{k} e\left(1,2, n, \mu_{1}, \ldots, \mu_{k}\right)
$$

Finally, the momentum label $p_{i}$ of each edge is uniquely defined by the position $i$ of the edge in the edge list.

Now that most properties of the Feynman integral are captured in the extended edge list and we have defined which edge list is smallest, we use McKay's canonicalisation algorithm [14] to efficiently rewrite the complete Feynman integral to canonical form. A simplified version of this algorithm is implemented in FORM code.

\subsection{Efficient tensor reduction}

It can be shown that the tensor reduction of ultraviolet and infrared subtraction terms, required for the $R^{*}$-operation, is equivalent to the tensor reduction of tensor vacuum bubble integrals. In general tensor vacuum integrals can be reduced to linear combinations of products of metric tensors $g^{\mu v}$ whose coefficients are scalar vacuum integrals. Specifically a rank $r$ tensor, $T^{\mu_{1} \ldots \mu_{r}}$, is written as a linear combination of $n=r ! / 2^{(r / 2)} /(r / 2)$ ! combinations of $r / 2$ metric tensors with coefficients $c_{\sigma}$, i.e.,

$$
T^{\mu_{1} \ldots \mu_{r}}=\sum_{\sigma \in 2} S_{r} c_{\sigma} T_{\sigma}^{\mu_{1} \ldots \mu_{r}}, \quad T_{\sigma}^{\mu_{1} \ldots \mu_{r}}=g^{\mu_{\sigma(1)} \mu_{\sigma(2)}} \ldots g^{\mu_{\sigma(r-1)} \mu_{\sigma(r)}} .
$$

Here we define ${ }_{2} S_{r}$ as the group of permutations which do not leave the tensor $T_{\sigma}^{\mu_{1} \ldots \mu_{r}}$ invariant. The coefficients $c_{\sigma}$ can be obtained by acting onto the tensor $T^{\mu_{1} \ldots \mu_{r}}$ with certain projectors $P_{\sigma}^{\mu_{1} \ldots \mu_{r}}$, such that

$$
c_{\sigma}=P_{\sigma}^{\mu_{1} \ldots \mu_{r}} T_{\mu_{1} \ldots \mu_{r}} .
$$

From this it follows that the orthogonality relation,

$$
P_{\sigma}^{\mu_{1} \ldots \mu_{r}} T_{\tau, \mu_{1} \ldots \mu_{r}}=\delta_{\sigma \tau}
$$

must hold, where $\delta$ is the Kronecker-delta. Since the projector $P_{\sigma}^{\mu_{1} \ldots \mu_{r}}$ of each tensor can also be written in terms of a linear combination of products of metric tensors, inverting an $n \times n$ matrix determines all the projectors. However, there are two issues. The first is that the size of the matrix grows rather rapidly as $r$ increases. Instead of solving an $n \times n$ linear system, the symmetry group of the metric tensors can be utilised to reduce the size of the system. From eq. (3.14) it follows that the projector $P_{\sigma}$ is in the same symmetry group (the group of permutations which leave it invariant) as $T_{\sigma}$. For example, given a permutation $\sigma_{1}=(123 \ldots(r-1) r)$,

$$
T_{\sigma_{1}}^{\mu_{1} \ldots \mu_{r}}=g^{\mu_{1} \mu_{2}} g^{\mu_{3} \mu_{4}} \ldots g^{\mu_{r-1} \mu_{r}} .
$$

The corresponding projector $P_{\sigma_{1}}^{\mu_{1} \ldots \mu_{r}}$ must be symmetric under interchanges of indices such as $\mu_{1} \leftrightarrow$ $\mu_{2},\left(\mu_{1}, \mu_{2}\right) \leftrightarrow\left(\mu_{3}, \mu_{4}\right)$ and so on. Grouping the metric tensors by the symmetry leads to the fact 
that $P_{\sigma}$ is actually written in a linear combination of a small number of $m$ tensors instead of $n$ $(m \leq n)$,

$$
P_{\sigma}^{\mu_{1} \ldots \mu_{r}}=\sum_{k=1}^{m} b_{k} \sum_{\tau \in A_{m}^{\sigma}} T_{\tau}^{\mu_{1} \ldots \mu_{r}}
$$

The set of groups $\left\{A_{k}^{\sigma} \mid k=1 . . m\right\}$ must therefore each be closed under the permutations which leaves $T_{\sigma}$ invariant and at the same time their union must cover once the group ${ }_{2} S_{n}$. Contracting $P_{\sigma}$ with a representative in each group gives an $m \times m$ matrix which can be inverted to yield the coefficients $b_{k}$. The number of unknowns $m$ is $m=5$ for $r=8$ and $m=22$ for $r=16$, whereas we have $n=105$ for $r=8$ and $n=2027025$ for $r=16$. The comparison of these numbers illustrates that the exploitation of the symmetry of the projectors makes it possible to find the tensor reduction even for very large values of $r$, which could never have been obtained by solving the $n \times n$ matrix.

The second issue with tensors of high rank is the large number of intermediate terms that are created. Even though the system for the projector can be solved efficiently, $\mathscr{O}\left(n^{2}\right)$ terms will be created, of which some will merge due to symmetry. Let us consider rank 6, with 15 terms:

$$
c_{1} g^{\mu_{1} \mu_{2}} g^{\mu_{3} \mu_{4}} g^{\mu_{5} \mu_{6}}+c_{2} g^{\mu_{1} \mu_{3}} g^{\mu_{2} \mu_{4}} g^{\mu_{5} \mu_{6}}+\ldots \text {. }
$$

In most practical situations there is symmetry, both on the inside of the object that will be projected as on the outside. For example,

$$
A\left(p_{1}^{\mu_{1}} p_{1}^{\mu_{2}} p_{1}^{\mu_{3}} p_{1}^{\mu_{4}} p_{2}^{\mu_{5}} p_{2}^{\mu_{6}}\right) p_{3}^{\mu_{1}} p_{3}^{\mu_{2}} p_{4}^{\mu_{3}} p_{4}^{\mu_{4}} p_{4}^{\mu_{5}} p_{4}^{\mu_{6}}
$$

is symmetric in exchanges of $\mu_{1}, \ldots, \mu_{4}$ and $\mu_{5}, \mu_{6}$ inside $A$, and is symmetric in $\mu_{1}, \mu_{2}$ and $\mu_{3}, \ldots, \mu_{6}$ outside $A$. The symmetry inside the object $A$ will enforce that coefficient $c_{1}$ and $c_{2}$ (and others) will actually be the same. The symmetry on the outside will cause terms to merge. In the end, we could have used the symmetrised variant of eq. (3.17) instead:

$$
c_{1} \cdot\left(g^{\mu_{1} \mu_{2}} g^{\mu_{3} \mu_{4}} g^{\mu_{5} \mu_{6}}+2 g^{\mu_{1} \mu_{3}} g^{\mu_{2} \mu_{4}} g^{\mu_{5} \mu_{6}}\right)+c_{3}\left(2 g^{\mu_{1} \mu_{2}} g^{\mu_{3} \mu_{5}} g^{\mu_{4} \mu_{6}}+10 g^{\mu_{1} \mu_{5}} g^{\mu_{2} \mu_{6}} g^{\mu_{3} \mu_{4}}\right) \text {. }
$$

We see that only two coefficients have to be computed instead of 15 and that there are only 4 terms in the output instead of 15 . The challenge is to prevent these terms from being created in the first place by exploiting symmetry, instead of starting from eq. (3.17). We make use of the optimised FORM command dd_, which creates the tensor structure $T^{\mu_{1}, \ldots, \mu_{r}}$ without generating duplicates. If we evaluate dd_ $(\mathrm{p} 1, \mathrm{p} 1, \mathrm{p} 1, \mathrm{p} 1, \mathrm{p} 2, \mathrm{p} 2)$ and strip the coefficient we get $\mathrm{p} 1 . \mathrm{p} 1^{\wedge} 2 \star \mathrm{p} 2 . \mathrm{p} 2+$ $\mathrm{p} 1 \cdot \mathrm{p} 1 * \mathrm{p} 1 \cdot \mathrm{p} 2 \wedge 2$. These two terms represent the structure outside of $c_{1}$ and $c_{3}$ in (3.19). For each of these two terms, we solve for the coefficient. Next, we recreate the metric structures that would give this specific contraction.

A term generated by dd_consists of two different factors: $(p \cdot p)^{a}$ and $\left(p_{1} \cdot p_{2}\right)^{a}$. For $(p \cdot p)^{a}$, we collect all possible indices involved with $p$. For eq. (3.18), this would be $\mu_{1}, \ldots, \mu_{4}$. Then we select all possible ways to get $2 a$ elements from that list with distrib_. Next, we use $d d_{-}$on those indices. Thus, for $p_{1} \cdot p_{1}$ in the example we would get $g^{\mu_{1} \mu_{2}}+g^{\mu_{1} \mu_{3}}+g^{\mu_{2} \mu_{3}}$. For cases such as $\left(p_{1} \cdot p_{2}\right)^{a}$, we select $a$ from the list of indices associated to $p_{1}$ and $a$ from the list of $p_{2}$. Then we permute over the list of $p_{2}$. Using this algorithm, one can generate all possible contractions from the result without generating duplicates. To apply the outside symmetry, one can easily fill in the outside momenta associated to the indices instead of the indices themselves. distrib_and dd_ will take the symmetry into account automatically. 


\section{Five-loop results}

In this section we briefly discuss the five-loop results for the beta function and the Higgs-boson decay to gluons. With the same setup, we have also computed the Higgs decay to bottom quarks and the electromagnetic $R$-ratio at five loops [12]. Since these were re-computations, see ref. [15] and ref. [16] and references therein, respectively, we will not address these results here.

\subsection{The beta function}

Using the method described above, we have computed the five-loop beta function of QCD (obtained before in ref. [17]) and its generalizations to a general compact gauge group in the standard $\overline{\mathrm{MS}}$ scheme, using the background field method in the Feynman gauge [11]. See refs. [18,19] for more recent calculations with gauge dependence. The analytic result in terms of rational numbers and the values $\zeta_{n}, n=3,4,5$ of the Riemann $\zeta$-function can be found in eq. (3.5) of ref. [11].

The $\overline{\text { MS }}$ result can be transformed to the MiniMOM (MM) scheme [20,21], which may be more convenient for extending analyses of the strong coupling constant into the non-perturbative regime, using the FORCER calculations of four-loop vertex functions in ref. [22]. For the gaugedependent general result see eq. (B.4) of ref. [22]. It is interesting to note, e.g., in the context of refs. $[23,24]^{2}$, that the MiniMOM beta function in the Landau gauge, unlike the $\overline{\mathrm{MS}}$ result, does not include $\zeta_{4}$. The same was observed for the beta function of QED in the MOM scheme in ref. [28]. For further discussions of the issue of the $\zeta$-function values, see ref. [29] and references therein.

The numerical expansion of the $\overline{\mathrm{MS}}$ beta function of QCD is very benign to five loops with

$$
\begin{aligned}
& \widetilde{\beta}\left(\alpha_{\mathrm{s}}, n_{f}=3\right)=1+0.565884 \alpha_{\mathrm{s}}+0.453014 \alpha_{\mathrm{s}}^{2}+0.676967 \alpha_{\mathrm{s}}^{3}+0.580928 \alpha_{\mathrm{s}}^{4}+\ldots, \\
& \widetilde{\beta}\left(\alpha_{\mathrm{s}}, n_{f}=4\right)=1+0.490197 \alpha_{\mathrm{s}}+0.308790 \alpha_{\mathrm{s}}^{2}+0.485901 \alpha_{\mathrm{s}}^{3}+0.280601 \alpha_{\mathrm{s}}^{4}+\ldots, \\
& \widetilde{\beta}\left(\alpha_{\mathrm{s}}, n_{f}=5\right)=1+0.401347 \alpha_{\mathrm{s}}+0.149427 \alpha_{\mathrm{s}}^{2}+0.317223 \alpha_{\mathrm{s}}^{3}+0.080921 \alpha_{\mathrm{s}}^{4}+\ldots
\end{aligned}
$$

for $\widetilde{\beta} \equiv-\beta\left(a_{\mathrm{s}}\right) /\left(a_{\mathrm{s}}^{2} \beta_{0}\right)$ with $\beta_{0}=11-2 / 3 n_{f}$ and $a_{\mathrm{s}}=\alpha_{\mathrm{s}} /(4 \pi)$. The five-loop (N $\left.{ }^{4} \mathrm{LO}\right)$ contribution changes the beta function by less than $1 \%$ at $\alpha_{\mathrm{s}}=0.47$ for $n_{f}=4$ and at $\alpha_{\mathrm{s}}=0.39$ for $n_{f}=3$ quark flavours. The $\mathrm{N}^{n \geq 2} \mathrm{LO}$ coefficients are larger in the Landau-gauge MiniMOM scheme,

$$
\begin{aligned}
& \widetilde{\beta}_{\mathrm{MM}}\left(\alpha_{\mathrm{s}}, n_{f}=3\right)=1+0.565884 \alpha_{\mathrm{s}}+0.941986 \alpha_{\mathrm{s}}^{2}+2.30450 \alpha_{\mathrm{s}}^{3}+6.64749 \alpha_{\mathrm{s}}^{4}+\ldots, \\
& \widetilde{\beta}_{\mathrm{MM}}\left(\alpha_{\mathrm{s}}, n_{f}=4\right)=1+0.490197 \alpha_{\mathrm{s}}+0.645215 \alpha_{\mathrm{s}}^{2}+1.63846 \alpha_{\mathrm{s}}^{3}+3.46687 \alpha_{\mathrm{s}}^{4}+\ldots \\
& \widetilde{\beta}_{\mathrm{MM}}\left(\alpha_{\mathrm{s}}, n_{f}=5\right)=1+0.401347 \alpha_{\mathrm{s}}+0.328852 \alpha_{\mathrm{s}}^{2}+1.02689 \alpha_{\mathrm{s}}^{3}+0.84177 \alpha_{\mathrm{s}}^{4}+\ldots
\end{aligned}
$$

where $\alpha_{\mathrm{s}} \equiv \alpha_{\mathrm{s}}^{\mathrm{MM}}$, and exhibit a definite growth with the order that is absent in the $\overline{\mathrm{MS}}$ case.

The different behaviour of the $\alpha_{\mathrm{s}}$-expansion of the beta function of QCD in these two schemes is illustrated in the upper part of fig. 1. At $\alpha_{\mathrm{s}}^{\mathrm{MM}}=0.25$, which corresponds to an $\overline{\mathrm{MS}}$ value of $\alpha_{\mathrm{s}}=0.2$ for $n_{f}=4$ at $\mathrm{N}^{4} \mathrm{LO}-$ for the conversion see eq. (B.2) of ref. [22] - the individual $\mathrm{N}^{2} \mathrm{LO}$, $\mathrm{N}^{3} \mathrm{LO}$ and $\mathrm{N}^{4} \mathrm{LO}$ contributions add $3.6 \%, 2.3 \%$ and $1.2 \%$, respectively, to the total NLO result.

\footnotetext{
${ }^{2}$ It appears that Euclidean physical quantities do not receive even- $n \zeta_{n}$, i.e., $\pi^{2}$, contributions in renormalization schemes, such as MiniMOM or the scheme suggested in ref. [25], in which the beta function is free of such terms. Beyond the cases covered in refs. [23, 24], this has also been established to $\mathrm{N}^{4} \mathrm{LO}$ [26] for the scheme-independent versions, see refs. [19,27] of all 11 propagator and vertex functions computed in ref. [22].
} 

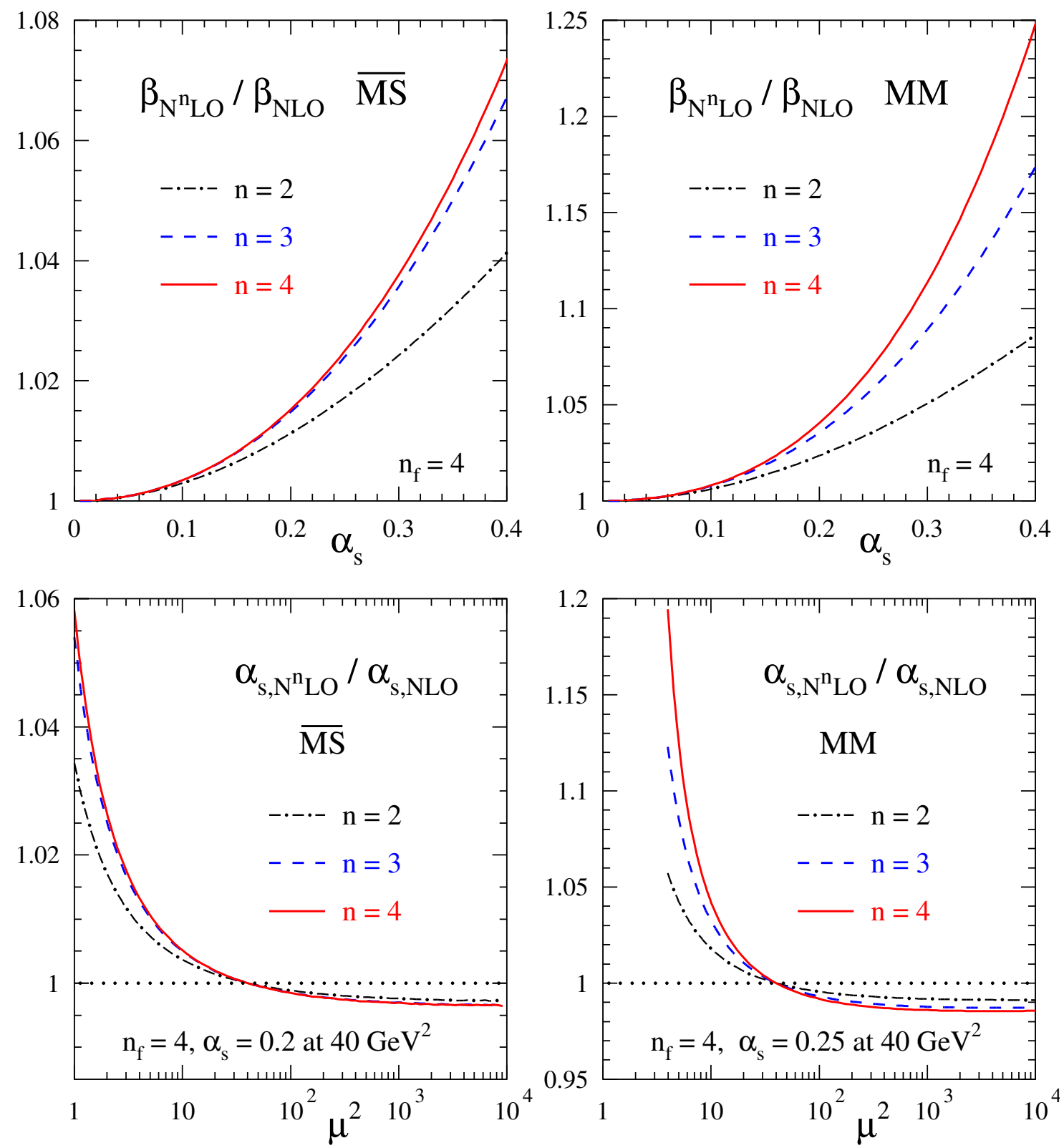

Figure 1: Upper panels: the $\mathrm{N}^{2} \mathrm{LO}, \mathrm{N}^{3} \mathrm{LO}$ and $\mathrm{N}^{4} \mathrm{LO}$ approximation to the beta function of QCD with four flavours in the $\overline{\mathrm{MS}}$ and MiniMOM (MM) schemes, normalized to their common NLO values. Lower panels: the resulting scale dependence of the respective coupling constants for order-independent reference values of 0.2 for $\overline{\mathrm{MS}}$ and 0.25 for $\mathrm{MM}$ at $\mu^{2}=40 \mathrm{GeV}^{2}$.

Unlike the $\overline{\mathrm{MS}}$ case, where the expansion appears to converge up to rather large values of $\alpha_{\mathrm{s}}$, the $\mathrm{N}^{3} \mathrm{LO}$ contribution exceeds the $\mathrm{N}^{2} \mathrm{LO}$ one for $\alpha_{\mathrm{s}} \geq 0.4$, and the $\mathrm{N}^{4} \mathrm{LO}$ effect that at $\mathrm{N}^{3} \mathrm{LO}$ for $\alpha_{\mathrm{s}}>0.47$.

Hence, as illustrated in the lower part of fig. 1, also the running of $\alpha_{\mathrm{s}}$ in the MiniMOM scheme becomes unstable at a scale of about $\mu \approx 2 \mathrm{GeV}$. For a comparison of the scale stability of the $R$-ratio in these two schemes at a moderate $\left(\alpha_{\mathrm{s}}\left(Q^{2}\right) \simeq 0.2\right.$ with $n_{f}=4$ in $\left.\overline{\mathrm{MS}}\right)$ and a small $\left(\alpha_{\mathrm{s}}\left(Q^{2}\right) \simeq\right.$ 0.3 and $\left.n_{f}=3\right)$ scale, see figs. 5 and 6 of ref. [12]. 

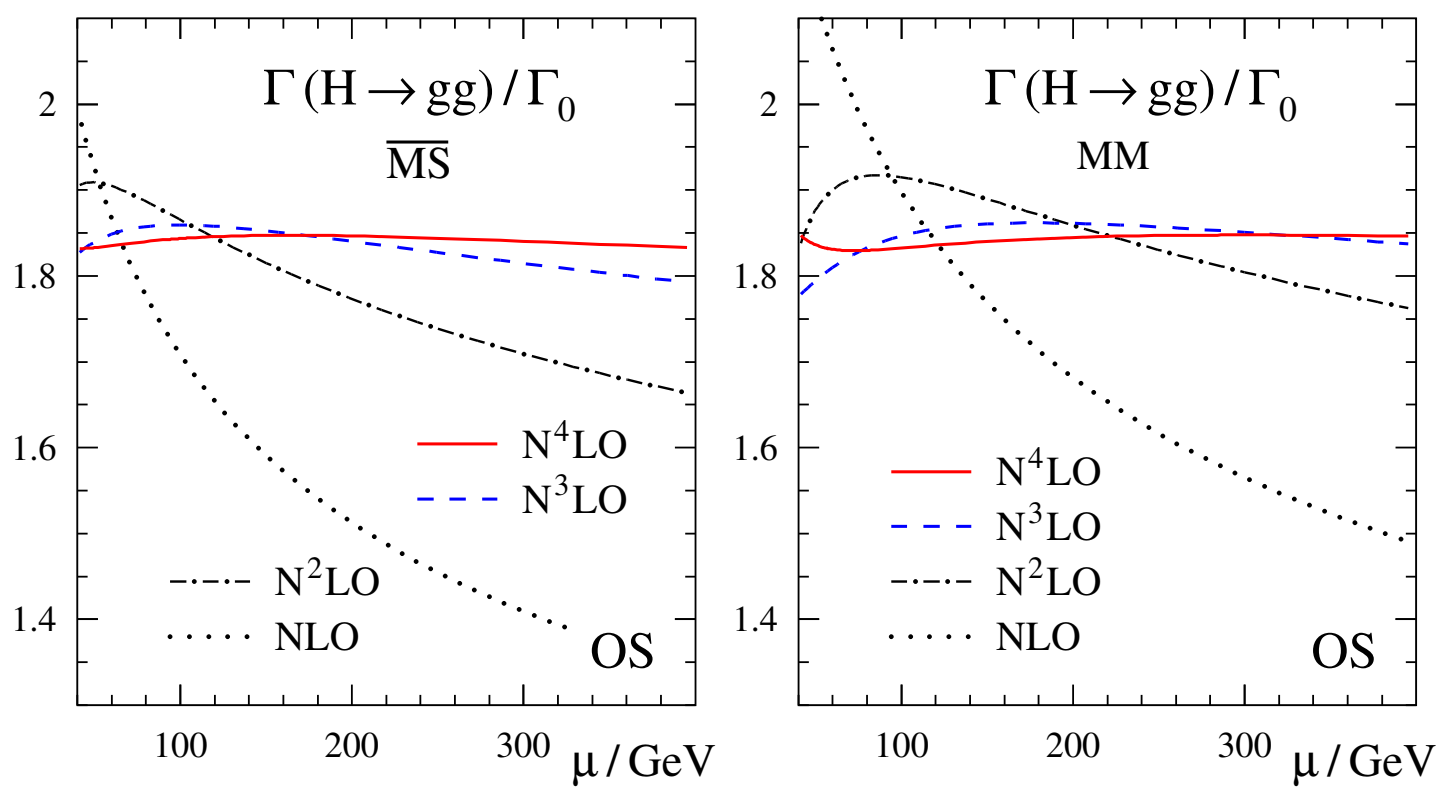

Figure 2: The renormalization scale dependence of $\Gamma_{H \rightarrow g g}$, normalized as described in the text, in the $\overline{\mathrm{MS}}$ and MiniMOM schemes, for $\alpha_{\mathrm{s}}\left(M_{Z}\right)=0.118$ in $\overline{\mathrm{MS}}, M_{H}=125 \mathrm{GeV}$ and an on-shell top-mass in $173 \mathrm{GeV}$.

\subsection{Higgs decay to gluons}

In the heavy-top limit, there is an effective coupling of the Higgs boson to gluons. If the light-quark masses are neglected the Higgs decay to hadrons can be expressed, using the optical theorem, as

$$
\Gamma_{H \rightarrow g g}=\frac{\sqrt{2} G_{\mathrm{F}}}{M_{H}}\left|C_{1}\right|^{2} \operatorname{Im} \Pi^{G G}\left(-M_{H}^{2}-i \delta\right) .
$$

The Wilson coefficient $C_{1}$, which includes the top-mass (scheme) dependence, is known to $\mathrm{N}^{4} \mathrm{LO}$, see ref. [30] and reference therein. Due to the analytical continuation from the spacelike case, only the pole part of Higgs-boson self-energy $\Pi^{G G}$ induced by the effective $\mathrm{Hgg}$ coupling is required:

$$
\operatorname{Im} \Pi^{G G}\left(-q^{2}-i \delta\right)=\sin (L \pi \varepsilon) \Pi^{G G}\left(q^{2}\right)=\Pi^{G G}\left(q^{2}\right) L \pi \varepsilon+\ldots,
$$

where $D=4-2 \varepsilon$ and $L$ is the number of loops. Consequently, $\operatorname{Im} \Pi^{G G}\left(-q^{2}-i \delta\right)$ and hence the $\mathrm{N}^{4} \mathrm{LO}$ decay rate can be computed using the setup described above. The computation is much more costly than that of the beta function and took almost two months on the machines available to us.

For an on-shell top mass of $173 \mathrm{GeV}$, the perturbative expansion of the decay width reads

$$
\Gamma_{H \rightarrow g g}^{\overline{\mathrm{MS}}}=\Gamma_{0}\left(1+5.703052 \alpha_{\mathrm{s}}+15.51204 \alpha_{\mathrm{s}}^{2}+12.6660 \alpha_{\mathrm{s}}^{3}-69.3287 \alpha_{\mathrm{s}}^{4}+\ldots\right)
$$

at $n_{f}=5$ for the renormalization scale $\mu=M_{H}$ with ( $G_{F}$ is the Fermi constant)

$$
\Gamma_{0}=G_{F} M_{H}^{3} /\left(36 \pi^{3} \sqrt{2}\right) \cdot\left(\alpha_{\mathrm{s}}\left(M_{H}^{2}\right)\right)^{2} .
$$

Note that the large ratio of the $\mathrm{N}^{4} \mathrm{LO}$ and $\mathrm{N}^{3} \mathrm{LO}$ coefficients is accidental, and not indicating a break-down of the perturbative expansion, see ref. [12]. The dependence of the numerical result on the scheme and value of $m_{\text {top }}$ is very weak. The corresponding MiniMOM result is given by

$$
\Gamma_{H \rightarrow g g}^{\mathrm{MM}}=\Gamma_{0}\left(1+4.345814 \alpha_{\mathrm{s}}+4.379443 \alpha_{\mathrm{s}}^{2}-21.5506 \alpha_{\mathrm{s}}^{3}-71.9231 \alpha_{\mathrm{s}}^{4}+\ldots\right)
$$


where, of course, $\alpha_{\mathrm{s}} \equiv \alpha_{\mathrm{s}}^{\mathrm{MM}}$ with $\alpha_{\mathrm{s}}^{\mathrm{MM}}\left(M_{Z}\right)=1.096 \alpha_{\mathrm{s}}\left(M_{Z}\right)$ for the standard $\overline{\mathrm{MS}}$ value of 0.118 . This higher value is compensated by lower-order coefficients in eqs. (4.7) and that are smaller than their $\overline{\mathrm{MS}}$ counterparts; The $\mathrm{N}^{3} \mathrm{LO}$ and $\mathrm{N}^{4} \mathrm{LO}$ terms for $n_{f}=5$ are not smaller in MiniMOM, though.

Taking into account also the renormalization scale dependence as shown in fig. 2, we arrive at

$$
\Gamma_{\mathrm{N}^{4} \mathrm{LO}}(H \rightarrow g g)=\Gamma_{0}\left(1.844 \pm 0.011_{\text {series }} \pm 0.045_{\alpha_{\mathrm{s}}}\left(M_{\mathrm{Z}}\right), 1 \%\right)
$$

with $\alpha_{\mathrm{s}}\left(M_{H}\right)=0.11264$, the $\overline{\mathrm{MS}}$ value corresponding to $\alpha_{\mathrm{s}}\left(M_{Z}\right)=0.118$, in eq. (4.6). For $1 / m_{\text {top }}$ corrections and light-quark mass effects, see ref. [31] and references therein.

\section{Summary and outlook}

We have sketched how the $R^{*}$-operation can be used to compute the poles of Feynman diagrams. Additionally, we have identified computational difficulties when performing five-loop calculations. We provide four solutions that reduce the number of terms that are generated.

Using these methods, we have computed the five-loop beta function for Yang-Mills theory with fermions [11], and the $R$-ratio and Higgs-boson decay widths $\Gamma_{H}$ to quarks and gluons [12]. We have briefly discussed the results for the beta function and for $\Gamma_{H \rightarrow g g}$ in the heavy-top limit. In the usual $\overline{\mathrm{MS}}$ scheme, the perturbative running of the strong coupling constant $\alpha_{\mathrm{s}}$ is now fully under control for all practical purposes. The uncertainty of $\Gamma_{H \rightarrow g g}$ due to the truncation of the perturbation series is now much smaller than that due to present uncertainty of the value of $\alpha_{\mathrm{s}}\left(M_{Z}\right)$; measurements of a comparable accuracy are not possible at the LHC, but may be in reach of a future $e^{+} e^{-}$collider.

It is possible to use our setup to extend recent calculations of splitting-function moments [32, 33 ] to five loops for very low moments $N$. The non-singlet splitting functions have already been computed in this manner at $N=2$ and $N=3$; the results will be presented and discussed elsewhere. The extension of these computations to $N \geq 4$ present a computational challenge well beyond that posed by $\Gamma_{H \rightarrow g g}$, the by far hardest case presented here.

\section{Acknowledgements}

We would like to thank K. Chetyrkin for useful discussions. The research reported here has been supported by the European Research Council (ERC) Advanced Grant 320651,HEPGAME, and the UK Science \& Technology Facilities Council (STFC) grant ST/L000431/1.

\section{References}

[1] K.G. Chetyrkin and F.V. Tkachov, Phys. Lett. B114 (1982) 340

[2] K.G. Chetyrkin and V.A. Smirnov, Phys. Lett. B144 (1984) 419

[3] F. Herzog and B. Ruijl, JHEP 05 (2017) 37, arXiv:1703.03776

[4] T. Ueda, B. Ruijl and J.A.M. Vermaseren, PoS LL2016 (2016) 070, arXiv:1607.07318

[5] B. Ruijl, T. Ueda and J.A.M. Vermaseren, https://doi.org/10.5281/zenodo.1043944

[6] B. Ruijl, T. Ueda and J.A.M. Vermaseren, arXiv:1704.06650 
[7] J.A.M. Vermaseren, math-ph/0010025

[8] M. Tentyukov and J.A.M. Vermaseren, Comput. Phys. Commun. 181 (2010) 1419, hep-ph/0702279

[9] J. Kuipers, T. Ueda, J.A.M. Vermaseren and J. Vollinga, Comput. Phys. Commun. 184 (2013) 1453, arXiv: 1203.6543

[10] B. Ruijl, T. Ueda and J. Vermaseren, arXiv:1707.06453

[11] F. Herzog, B. Ruijl, T. Ueda, J.A.M. Vermaseren and A. Vogt, JHEP 02 (2017) 090, arXiv:1701.01404

[12] F. Herzog, B. Ruijl, T. Ueda, J.A.M. Vermaseren and A. Vogt, JHEP 08 (2017) 113, arXiv:1707.01044

[13] A.A. Vladimirov, Theor. Math. Phys. 43 (1980) 417

[14] B.D. McKay, Congressus Numerantium 30 (1981) 45

[15] P.A. Baikov, K.G. Chetyrkin and J.H. Kühn, Phys. Rev. Lett. 96 (2006) 012003, hep-ph/0511063

[16] P.A. Baikov, K.G. Chetyrkin, J.H. Kühn and J. Rittinger, Phys. Rev. Lett. 108 (2012) 222003, arXiv:1201.5804

[17] P.A. Baikov, K.G. Chetyrkin and J.H. Kühn, Phys. Rev. Lett. 118 (2017) 082002, arXiv:1606.08659

[18] T. Luthe, A. Maier, P. Marquard and Y. Schröder, JHEP 10 (2017) 166arXiv:1709.07718

[19] K.G. Chetyrkin, G. Falcioni, F. Herzog and J. Vermaseren, JHEP 10 (2017) 179, arXiv:1709.08541

[20] L. von Smekal, K. Maltman and A. Sternbeck, Phys. Lett. B681 (2009) 336, arXiv:0903.1696

[21] J.A. Gracey, J. Phys. A46 (2013) 225403, arXiv:1304.5347

[22] B. Ruijl, T. Ueda, J.A.M. Vermaseren and A. Vogt, JHEP 06 (2017) 040, arXiv:1703.08532

[23] M. Jamin and R. Miravitllas, arXiv:1711.00787

[24] J. Davies and A. Vogt, Phys. Lett. B776 (2018) 189, arXiv:1711.05267

[25] D. Boito, M. Jamin and R. Miravitllas, Phys. Rev. Lett. 117 (2016) 152001, arXiv:1606.06175

[26] K.G. Chetyrkin, private communication

[27] K.G. Chetyrkin, Nucl. Phys. B710 (2005) 499, hep-ph/0405193

[28] P.A. Baikov, K.G. Chetyrkin, J.H. Kühn and J. Rittinger, JHEP 07 (2012) 017, arXiv:1206.1284

[29] P.A. Baikov, K.G. Chetyrkin and J.H. Kühn, JHEP 04 (2017) 119, arXiv:1702.01458

[30] K. Chetyrkin, P. Baikov and J. Kühn, PoS LL2016 (2016) 010

[31] J. Davies, M. Steinhauser and D. Wellmann, Nucl. Phys. B920 (2017) 20, arXiv:1703.02988

[32] B. Ruijl, T. Ueda, J. Vermaseren, J. Davies and A. Vogt, PoS LL 2016 (2016) 071arXiv:1605.08408

[33] S. Moch, B. Ruijl, T. Ueda, J.A.M. Vermaseren and A. Vogt, JHEP 10 (2017) 041, arXiv:1707.08315 